\title{
COMBINATORIAL RICCI CURVATURE ON CELL-COMPLEX AND GAUSS-BONNNET THEOREM
}

\author{
KAZUYOSHI WATANABE
}

\begin{abstract}
In this paper, we introduce a new definition of the Ricci curvature on cell-complexes and prove the Gauss-Bonnnet type theorem for graphs and 2-complexes that decompose closed surfaces. The defferential forms on a cell complex is defined as linear maps on the chain complex, and the Laplacian operates this defferential forms. Our the Ricci curvature is defined by the combinatorial BochnerWeitzenböck formula. We prove some propositionerties of combinatorial vector fields on a cell complex.
\end{abstract}

\section{INTRODUCTION}

In this paper, we introduce a new definition of the Ricci curvature on cell-complexes and prove the Gauss-Bonnnet type theorem for graphs and 2-complexes that decompose closed surfaces. In the Riemanian geometry, the curvature plays an important role, and there are many results on the curvature on smooth manifolds. Especially the Gauss-Bonnet theorem is known as a fundamental propositionerty of a smooth closed manifold. The curvature on a cell complex was stuied in many ways. R. Forman defined the Ricci curvature on a cell complex with the Bochner-Weitzenböck formula on the cochain. With this curvature he also showed the Bochner's theorem, the Myers' theorem and so on. For the curvature defined by angles, Mccorollarymick Paul [7] established the Gauss-Bonnet theorem, but in the Forman's way the Gauss-Bonnet theorem does not hold.

R. Forman established the discrete Morse theory in [4]. He studied the function on a cell complex and the relation between critical cells and the homology of the cell complex. He also extended this thoery to the discrete Novikov-Morse theory, and in this theory he defined a defferential form on the cell complex. This defferential form is not the cochain of the cell complex but a linear map on the chain of the cell complex. In this paper we use this defferential forms to define the Ricci curvature. We introduce the $L^{2}$ inner product on the space of combinatorial defferential forms, and this inner product determines the Laplacian on combinatorial defferential forms. Then the Ricci curvature is definied with the combinatorial Bochner-Weitzenböck formula for the combinatorial defferential forms.

For the construction of the Bochner-Weitzenböck formula, we need the covariant of a 1-form. For this definition we present the 0 - and 2-neighbor vector. These vectors is roughly said "the pararell vectors". We define the covariant of a 1-form as the defference between the components of pararell vectors. Then for the cell complex with constant weights, the Ricci curvature is calculated as combinatorial computation,

$$
\operatorname{Ric}(\omega)(\tau>\sigma)=(2-\#\{0-\text { neighbor vector of }(\tau>\sigma)\})\left(\omega_{\sigma}^{\tau}\right)^{2} .
$$

This formula means that the Ricci curvature on the cell complex at the cell $\sigma$ is determined by the constructure around $\sigma$. For a graph or a 2-dimensional complex that decomposes a closed surface, the Ricci curvature for a unit vector at a vertex (resp. at a face $f$ ) is independent of the choice of the unit vector, and we define this value as the Gauss curvature $g_{v}$ (resp. $g_{f}$ ) at the vertex $v$ (resp. the face $f$ ). We have the following Gauss-Bonnet type theorems.

2010 Mathematics Subject Classification. Primary 05E45, Secondary 53B21. 
TheOREM 1.1. Let $G$ be a finite simple graph. Then we have

$$
\sum_{v} g_{v}=2 \chi(G)
$$

where the sum is taken over all vertexes $v$ and $\chi(G)$ is the Euler number of $G$.

TheOREM 1.2. Let $M$ be a 2-dimensional quasiconvex cell complex that decomposes a 2-dimensional closed smooth surface. Then we have

$$
\sum_{v} g_{v}+\sum_{f} g_{f}=4 \chi(M)
$$

where the sums are taken over all vertexes $v$ and all faces $f$ respectivity, and $\chi(M)$ is the Euler number of $M$.

We also present the vector field on a cell complex. This is defined as a dual of a combinatorial defferential 1-form and we prove some propositionerties of this vector field.

\section{Combinatorial Defferential Forms}

2.1. Definition of Combinatorial Defferential Forms. In this section, we present a defferential form on a cell-complex intoruduced in [2]. Let $M$ be a regular cell complex of dimension $n$, and

$$
0 \longrightarrow C_{n}(M) \stackrel{\partial}{\longrightarrow} C_{n-1}(M) \stackrel{\partial}{\longrightarrow} \cdots \stackrel{\partial}{\longrightarrow} C_{0}(M) \longrightarrow 0
$$

be the real cellular chain complex of $M$. We set

$$
C_{*}(M)=\bigoplus_{p} C_{p}(M) .
$$

A linear map $\omega: C_{*}(M) \rightarrow C_{*}(M)$ is said to be of degree $d$ if for all $p=1, \ldots, n$,

$$
\omega\left(C_{p}(M)\right) \subset C_{p-d}(M) .
$$

We say that a linear map $\omega$ of degree $d$ is local if, for each $p$ and each oriented $p$-cell $\alpha, \omega(\alpha)$ is a linear combination of oriented $(p-d)$-cells that are faces of $\alpha$.

Definition 2.1. For $d \geq 0$, we say that a local linear map $\omega: C_{*}(M) \rightarrow C_{*}(M)$ of degree $d$ is a conbinatorial defferential $d$-form, and we denote the space of combinatorial differential $d$-forms by $\Omega^{d}(M)$.

We define the differential of combinatorial defferential forms

$$
d: \Omega^{d}(M) \rightarrow \Omega^{d+1}(M)
$$

as follows. For any $\omega \in \Omega^{d}(M)$ and any $p$-chain $c$, we define $(d \omega)(c) \in C_{p-(d+1)}(M)$ by

$$
(d \omega)(c)=\partial(\omega(c))-(-1)^{d} \omega(\partial c) .
$$

That is,

$$
d \omega=\partial \circ \omega-(-1)^{d} \omega \circ \partial .
$$

LEMma 2.2 ([2]). The differential for combinatorial defferential forms satisfies the following propositionerties.

- $d\left(\Omega^{d}(M)\right) \subseteq \Omega^{(d+1)}(M)$.

- $d^{2}=0$. 
This lemma determines the differential complex

$$
\Omega^{*}(M): 0 \longrightarrow \Omega^{0}(M) \stackrel{d}{\longrightarrow} \Omega^{1}(M) \stackrel{d}{\longrightarrow} \cdots \stackrel{d}{\longrightarrow} \Omega^{n}(M) \longrightarrow 0 .
$$

THEOREM 2.3 ([2]). The cohomology of this complex is isomorphic to the singular cohomology of $M$. That is,

$$
H^{*}\left(\Omega^{*}(M)\right) \cong H^{*}(M) .
$$

2.2. Laplacian for Combinatorial Defferential Forms. Let us define an inner product on $C_{*}(M)$. For any two $p$-cells $\sigma, \sigma^{\prime}$, we set an innner product as

$$
\left\langle\sigma, \sigma^{\prime}\right\rangle=\delta_{\sigma, \sigma^{\prime}} w_{\sigma},
$$

where $\delta_{\sigma, \sigma^{\prime}}$ is the Kronecker's delta, that is, $\delta_{\sigma, \sigma^{\prime}}=1$ for $\sigma=\sigma^{\prime}$ and the others are 0 , and $w_{\sigma}>0$ is a weight of a cell $\sigma$. We define the $L^{2}$ inner product for combinatorial differential forms. For two $d$-forms $u, v$, we set

$$
\langle u, v\rangle=\sum_{\sigma} \frac{1}{w_{\sigma}}\langle u(\sigma), v(\sigma)\rangle,
$$

where the sum is taken over all cells $\sigma$ in $M$.

Let us consider the adjecent operator of differential with respect to the inner product,

$$
d^{*}: \Omega^{d}(M) \rightarrow \Omega^{d-1}(M) .
$$

That is, for a $d$-form $u$ and a $(d-1)$-form $v$ we have

$$
\left\langle d^{*} u, v\right\rangle=\langle u, d v\rangle
$$

The space of combinatorial differential $d$-forms $\Omega^{d}(M)$ is a sub vector space of the space of linear maps of degree $d$ on the chain $C_{*}(M)$. Then we set $p$ as the projection on the space of linear maps of degree $d$ on the chain $C_{*}(M)$ to the space of combinatorial differential $d$-forms $\Omega^{d}(M)$.

LEMMA 2.4. For any d-form $\omega$, we have

$$
d^{*}=p \circ\left(\partial^{*} \circ \omega-(-1)^{(d-1)} \omega \circ \partial^{*}\right) .
$$

Proof. For a $p$-dimensional cell $\tau$ and a $(p-d)$-dimensional cell $\sigma$ which is a face of $\tau$, we put a $d$-form $e_{\sigma}^{\tau}$ such that

$$
e_{\sigma}^{\tau}(\tau)=\sigma
$$

and the value is 0 for other cells. They form a basis of $d$-forms as a real vector space.

Let $\tau, \sigma, \alpha, \beta$ be cells, and assume that $e_{\sigma}^{\tau}$ is a $d$-form and $e_{\beta}^{\alpha}$ is a $(d-1)$-form. Then we have

$$
\begin{aligned}
\left\langle d^{*} e_{\sigma}^{\tau}, e_{\beta}^{\alpha}\right\rangle & =\left\langle e_{\sigma}^{\tau}, d e_{\beta}^{\alpha}\right\rangle \\
& =\left\langle e_{\sigma}^{\tau}, \partial \circ e_{\beta}^{\alpha}\right\rangle-(-1)^{(d-1)}\left\langle e_{\sigma}^{\tau}, e_{\beta}^{\alpha} \circ \partial\right\rangle .
\end{aligned}
$$

We put $A$ as the right-hand side of equation (2.11), and have

$$
\left\langle A e_{\sigma}^{\tau}, e_{\beta}^{\alpha}\right\rangle=\left\langle e_{\sigma}^{\tau}, \partial e_{\beta}^{\alpha}\right\rangle-\underset{3}{(-1)^{(d-1)}}\left\langle e_{\sigma}^{\tau} \circ \partial^{*}, e_{\beta}^{\alpha}\right\rangle .
$$


Now we calculate the last term of equation (2.13),

$$
\begin{aligned}
\left\langle e_{\sigma}^{\tau}, e_{\beta}^{\alpha} \circ \partial\right\rangle & =\sum_{c: \text { cell }} \frac{1}{w_{c}}\left\langle e_{\sigma}^{\tau}(c), e_{\beta}^{\alpha}(\partial c)\right\rangle \\
& =\frac{1}{w_{c}}\left\langle\sigma, e_{\beta}^{\alpha}(\partial \tau)\right\rangle \\
& =\left\{\begin{array}{lr}
\frac{w_{\sigma}}{w_{\tau}}(-1)^{\tau>\alpha} & \text { for } \tau>\alpha, \sigma=\beta \\
0 & \text { otherwise. }
\end{array}\right.
\end{aligned}
$$

We calculate the last term of eqation (2.14),

$$
\begin{aligned}
\left\langle e_{\sigma}^{\tau} \circ \partial^{*}, e_{\beta}^{\alpha}\right\rangle & =\sum_{c: \text { cell }} \frac{1}{w_{c}}\left\langle e_{\sigma}^{\tau}\left(\partial^{*} c\right), e_{\beta}^{\alpha}(c)\right\rangle \\
& =\frac{1}{w_{\alpha}}\left\langle e_{\sigma}^{\tau}\left(\partial^{*} \alpha\right), \beta\right\rangle \\
& =\left\{\begin{array}{lr}
\frac{w_{\sigma}}{w_{\tau}}(-1)^{\tau>\alpha} & \text { for } \tau>\alpha, \sigma=\beta \\
0 & \text { otherwise. }
\end{array}\right.
\end{aligned}
$$

Then we have

$$
d^{*} e_{\sigma}^{\tau}=A e_{\sigma}^{\tau}
$$

DEFINITION 2.5. We define the Laplacian for combinatorial defferential forms by

$$
\Delta=d d^{*}+d^{*} d .
$$

Theorem 2.6. Let $M$ be a finite regular cell-complex. Then we have

$$
\operatorname{Ker}(\Delta) \cong H^{*}\left(\Omega^{*}(M)\right) \cong H^{*}(M) .
$$

Proof. The Laplacian is a self-adjoint operator on the finite dimensional vector space $\Omega^{*}(M)$ and we have $\operatorname{Ker}(\Delta)=\operatorname{Ker}(d) \cap \operatorname{Ker}\left(d^{*}\right)$. We consider a map

$$
\begin{aligned}
\operatorname{Ker}(\Delta) & \rightarrow H^{*}\left(\Omega^{*}(M)\right) \\
u & \mapsto[u] .
\end{aligned}
$$

This map is well-defined and injective. Next we prove that this map is surjective. The Laplacian has an eigen decomposition, and we denote the eigen values of the Laplacian by $\lambda_{0}, \ldots, \lambda_{k}$ are eigen values. Let $u$ be a closed form. Then we have an eigen decomposition for the Laplacian

$$
\Delta u=\sum_{i=1, \ldots, k} \lambda_{i} u_{i}
$$

where $u_{i}$ are eigen vectors for $\lambda_{i}$ respectively such that $u=\sum_{i=0, \ldots, k} u_{i}$. Then putting

$$
u^{\prime}=\sum_{i=1, \ldots, k} \frac{d^{*} u_{i}}{\lambda_{i}}
$$

we have

$$
u=u_{0}+d u^{\prime}
$$

We conclude that the map 2.20) is an isomorphism. 
2.3. Combinatorial function on cell-complex. We realize a combinatorial 0-form as a function. We set $f \in \Omega^{0}(M)$, that is,

$$
f: C^{*}(M) \rightarrow C^{*}(M)
$$

For any cell $\sigma$, we have

$$
f(\sigma)=f_{\sigma} \sigma
$$

and we realize $f_{\sigma} \in \mathbf{R}$ as the value of the function $f$. For a $p$-dimensional cell $\tau$, the derivative of $f$ is

$$
d f(\tau)=\sum_{\sigma: \tau>\sigma}(f(\tau)-f(\sigma))(-1)^{\tau>\sigma} \sigma,
$$

where the sum is taken over all $(p-1)$-dimensional cells $\sigma$ that are faces of $\tau$, and $(-1)^{\tau>\sigma}$ is the incidence number between $\tau$ and $\sigma$.

LEMMA 2.7. Let $M$ be a regular cell-complex and $f$ a function on $M$, where we identify $M$ with the set of cells of $M . f$ is locally constant if and only if $d f=0$.

2.4. Combinatorial 1-form on cell-complex. Let $\omega \in \Omega^{1}(M)$ be a combinatorial 1-form. For a $p$-dimensional cell $\tau$, we set

$$
\omega(\tau)=\sum_{\sigma: \tau>\sigma} \omega_{\sigma}^{\tau}(-1)^{\tau>\sigma} \sigma
$$

where the sum is taken over all $(p-1)$-dimensional cells $\sigma$ that are faces of $\tau$, and $(-1)^{\tau>\sigma}$ is the incidence number between $\tau$ and $\sigma$. We call the pair $(\tau>\sigma)$ a vector provided that a $p$-dimensional cell $\sigma$ is a face of $(p+1)$-dimensional cell $\tau$. We say that $\omega$ has the value $\omega_{\sigma}^{\tau}$ at the vector $(\tau>\sigma)$. For a $p$-dimensional cell $\mu$, the derivative of $\omega$ is

$$
d \omega(\mu)=\sum_{\left(\mu>\tau, \tau^{\prime}>\sigma\right)}\left(\omega_{\tau}^{\mu}+\omega_{\sigma}^{\tau}-\omega_{\tau^{\prime}}^{\mu}-\omega_{\sigma}^{\tau^{\prime}}\right)(-1)^{\mu>\tau}(-1)^{\tau>\sigma} \sigma,
$$

where the sum is taken over all two $(p-1)$-dimensional cells $\tau, \tau^{\prime}$ and $(p-2)$-dimensional cells $\sigma$ such that

$$
\mu>\tau>\sigma, \mu>\tau^{\prime}>\sigma, \tau \neq \tau^{\prime} .
$$

PROPOSITION 2.8. For a combinatorial 1-form $\omega$, we have $d \omega=0$ if and only if

$$
\omega_{\tau}^{\mu}+\omega_{\sigma}^{\tau}=\omega_{\tau^{\prime}}^{\mu}+\omega_{\sigma}^{\tau^{\prime}}
$$

for any $p$-dimensional cell $\mu$, any two $(p-1)$ dimensional cells $\tau, \tau^{\prime}$ and any $(p-2)$-dimensional cell $\sigma$ such that

$$
\mu>\tau>\sigma, \mu>\tau^{\prime}>\sigma, \tau \neq \tau^{\prime}
$$

For any cell $\sigma$, the dual derrivative of $\omega$ is

$$
d^{*} \omega(\sigma)=\left(-\sum_{\tau: \tau>\sigma} \frac{w_{\sigma}}{w_{\tau}} \omega_{\sigma}^{\tau}+\sum_{\rho: \rho<\sigma} \frac{w_{\sigma}}{w_{\rho}} \omega_{\rho}^{\sigma}\right) \sigma,
$$

where the first sum is taken over all ( $p+1)$-dimensional cells $\tau$ that have $\sigma$ as a face, and the second sum is over all $(p-1)$-dimensional cells $\rho$ that are the faces of $\sigma$. 


\section{Combinatorial Vector field on CELL-COMPleX}

Definition 3.1. We call a linear map $X: C_{*}(M) \rightarrow C_{(*+1)}(M)$ a combinatorial vector field on a cell-complex $M$ provided that for any cell $\sigma$ any component of $X(\sigma)$ has $\sigma$ as a face.

In the same manner as a 1 -form, for a $p$-dimensional cell $\sigma$ we set

$$
X(\sigma)=\sum_{\tau: \tau>\sigma} X_{\sigma}^{\tau}(-1)^{\tau>\sigma} \tau,
$$

where the sum is taken over all $(p-1)$-dimensional cells $\tau$ that have $\sigma$ as a face, and $(-1)^{\tau>\sigma}$ is the incidence number between $\tau$ and $\sigma$.

For a 1-form $\omega$ and a vector field $X$ on $M$, we define the pairing

$$
\omega(X)(\sigma)=\sum_{\tau: \tau>\sigma} \omega_{\sigma}^{\tau} X_{\sigma}^{\tau}
$$

Then for a function $f$ on $M$, we define

$$
X(f)(\sigma)=d f(X)(\sigma)=\sum_{\tau: \tau>\sigma} X_{\sigma}^{\tau}(f(\tau)-f(\sigma)) .
$$

Definition 3.2. Let $f$ be a function on $M$. We define the gradient vector field $\operatorname{grad}(f)$ of $f$ by

$$
\operatorname{grad}(f)_{\sigma}^{\tau}=\frac{w_{\sigma}}{w_{\tau}}(f(\tau)-f(\sigma)) .
$$

Let $X$ be a vector field on $M$. We also define the divergence $\operatorname{div}(X)$ of $f$ by

$$
\operatorname{div}(X)(\sigma)=-\sum_{\tau^{(p+1)}: \tau>\sigma} X_{\sigma}^{\tau}+\sum_{\rho^{(p-1)}: \rho>\sigma} X_{\rho}^{\sigma}
$$

We define the inner product for vector fields in the same manner as combinatorial defferential forms, i.e. for two vector fields $X, Y$

$$
\langle X, Y\rangle(\sigma)=\frac{1}{w_{\sigma}}\langle X(\sigma), Y(\sigma)\rangle=\sum_{\tau: \tau>\sigma} \frac{w_{\tau}}{w_{\sigma}} X_{\sigma}^{\tau} Y_{\sigma}^{\tau} .
$$

Then we have

$$
d f(X)=\langle X, \operatorname{grad}(f)\rangle .
$$

Definition 3.3. For a function $f$ on $M$, we define the integral of $f$ over $M$ by

$$
\int_{M} f=\sum_{\sigma} f(\sigma)
$$

where the sum is taken over all cells of $M$.

THEOREM 3.4. We assume that $M$ is a finite regular cell-complex. Let $f$ be a function on $M$ and $X$ a vector fieldon $M$. Then we have

$$
\int_{M}\langle\operatorname{grad}(f), X\rangle=\int_{6} f \operatorname{div}(X) .
$$


Proof. For a cell $\sigma$, we have

$$
\begin{aligned}
\sum_{\sigma}\langle\operatorname{grad}(f), X\rangle(\sigma) & =\sum_{\sigma} \sum_{\tau: \tau>\sigma} \frac{w_{\tau}}{w_{\sigma}} X_{\sigma}^{\tau} \cdot \frac{w_{\sigma}}{w_{\tau}}(f(\tau)-f(\sigma)) \\
& =\sum_{(\tau>\sigma)} X_{\sigma}^{\tau}(f(\tau)-f(\sigma)) \\
& =\sum_{\sigma} f(\sigma)\left(-\sum_{\tau^{(p+1)}: \tau>\sigma} X_{\sigma}^{\tau}+\sum_{\rho^{(p-1): \rho>\sigma}} X_{\rho}^{\sigma}\right) \\
& =\int_{M} f \operatorname{div}(X) .
\end{aligned}
$$

COROLlaRY 3.5. For any vector field $X$ on $M$ we have

$$
\int_{M} \operatorname{div}(X)=0
$$

Proof. For a constant function $f$, the gradient of $f$ vanishes. Then we take a constant function $f$ as

$$
f(\sigma)=1
$$

for any cell $\sigma$. Then we have the corollary from Theorem 3.4

COROLLARY 3.6. For any function $f$ on $M$ we have

$$
\int_{M} \Delta f=0
$$

Proof. For any cell $\sigma$,

$$
\begin{aligned}
\Delta f(\sigma) & =\left(-\sum_{\tau^{(p+1)}: \tau>\sigma}(f(\tau)-f(\sigma))+\sum_{\rho^{(p-1)}: \rho>\sigma}(f(\sigma)-f(\rho))\right) \\
& =\operatorname{div}(\operatorname{grad}(f))(\sigma) .
\end{aligned}
$$

Then we have the corollaryorally from the previous corollary.

\section{Combinatorial Ricci curvature}

\subsection{Combinatorial Ricci curvature.}

Definition 4.1. Let $M$ be a regular cell complex. We say that $M$ is quasiconvex if for every two distinct $(p+1)$-cells $\tau_{1}$ and $\tau_{2}$ of $M$, if $\overline{\tau_{1}} \cap \overline{\tau_{2}}$ contains a $p$-cell $\sigma$, then $\overline{\tau_{1}} \cap \overline{\tau_{2}}=\bar{\sigma}$. In particular this implies that $\overline{\tau_{1}} \cap \overline{\tau_{2}}$ contains at most one $p$-cell.

Let $M$ be a regular quasiconvex cell complex.

Definition 4.2. Let $\tau, \sigma$ be two cells of $M$ such that the dimension is $(p+1)$ and $p$ respectivity and $\sigma$ is a face of $\tau$.

We define a 0-neighbor vector of $(\tau>\sigma)$ as the following.

- The vectors $\left(\tau^{\prime}>\sigma\right)$ for $(p+1)$-cells $\tau^{\prime} \neq \tau$ such that there are no $(p+2)$-cell $\mu$ such that $\mu>\tau, \tau^{\prime}$.

- The vectors $\left(\tau>\sigma^{\prime}\right)$ for $p$-cells $\sigma^{\prime} \neq \sigma$ such that there are no $(p-1)$-cell $\rho$ such that $\sigma, \sigma^{\prime}>\rho$.

We define a 2-neighbor vector of $(\tau>\sigma)$ as the following.

- The vectors $\left(\mu>\tau^{\prime}\right)$ for $(p+1),(p+2)$-cells $\tau^{\prime}$ and $\mu$ such that $\mu>\tau>\sigma, \mu>\tau^{\prime}>\sigma$ and $\tau \neq \tau^{\prime}$. 
- The vectors $\left(\sigma^{\prime}>\rho\right)$ for $(p-1), p$-cells $\rho$ and $\sigma^{\prime}$ such that $\tau>\sigma>\rho, \tau>\sigma^{\prime}>\rho$ and $\sigma \neq \sigma^{\prime}$.

Definition 4.3. For a combinatorial 1-form $\omega$ on $M$, we define the combinatorial covariant derivative as

$$
\begin{aligned}
|\nabla \omega|^{2}(\tau>\sigma) & =\sum_{\left(\mu>\tau^{\prime}\right) ; 2-\text { neighbor }} \frac{w_{\sigma}}{w_{\mu}}\left(\omega_{\sigma}^{\tau}-\omega_{\tau^{\prime}}^{\mu}\right)^{2}+\sum_{\left(\sigma^{\prime}>\rho\right) ; 2-\text { neighbor }} \frac{w_{\rho}}{\tau}\left(\omega_{\sigma}^{\tau}-\omega_{\rho}^{\sigma^{\prime}}\right)^{2} \\
& +\sum_{\left(\tau^{\prime}>\sigma\right) ; 0-\text { neighbor }} \frac{\left(w_{\sigma}\right)^{2}}{w_{\tau} w_{\tau^{\prime}}}\left(\omega_{\sigma}^{\tau}+\omega_{\sigma}^{\tau^{\prime}}\right)^{2}+\sum_{\left(\tau>\sigma^{\prime}\right) ; 0-\text { neighbor }} \frac{w_{\sigma} w_{\sigma^{\prime}}}{\left(w_{\tau}\right)^{2}}\left(\omega_{\sigma}^{\tau}+\omega_{\sigma^{\prime}}^{\tau}\right)^{2},
\end{aligned}
$$

where the sums are taken over all 2-neighbor vectors and 0-neighbor vectors for $(\tau>\sigma)$ respectively.

Definition 4.4. For a combinatorial 1-form $\omega$ on $M$, we define the Laplacian of $|\omega|^{2}$ as

$$
\begin{array}{r}
\Delta^{b}|\omega|^{2}(\tau>\sigma)=\sum_{\left(\mu>\tau^{\prime}\right) ; 2-\text { neighbor }} \frac{w_{\sigma}}{w_{\mu}}\left(\left(\omega_{\sigma}^{\tau}\right)^{2}-\left(\omega_{\tau^{\prime}}^{\mu}\right)^{2}\right)+\sum_{\left(\sigma^{\prime}>\rho\right) ; 2-\text { neighbor }} \frac{w_{\rho}}{\tau}\left(\left(\omega_{\sigma}^{\tau}\right)^{2}-\left(\omega_{\rho}^{\sigma^{\prime}}\right)^{2}\right) \\
\quad+\sum_{\left(\tau^{\prime}>\sigma\right) ; 0-\text { neighbor }} \frac{\left(w_{\sigma}\right)^{2}}{w_{\tau} w_{\tau^{\prime}}}\left(\left(\omega_{\sigma}^{\tau}\right)^{2}+\left(\omega_{\sigma}^{\tau^{\prime}}\right)^{2}\right)+\sum_{\left(\tau>\sigma^{\prime}\right) ; 0-\text { neighbor }} \frac{w_{\sigma} w_{\sigma^{\prime}}}{\left(w_{\tau}\right)^{2}}\left(\left(\omega_{\sigma}^{\tau}\right)^{2}+\left(\omega_{\sigma^{\prime}}^{\tau}\right)^{2}\right),
\end{array}
$$

where the sums are taken over all 2-neighbor vectors and 0-neighbor vectors for $(\tau>\sigma)$ respectively.

This Laplacian is symmetry for vectors, hence we have

$$
\sum_{(\tau>\sigma)} \Delta^{b}|\omega|^{2}(\tau>\sigma)=0
$$

where the sum is taken over all vectors.

Definition 4.5. For a combinatorial 1-form $\omega$, we define the Ricci curvature on a vector $(\tau>\sigma)$ as

$$
\operatorname{Ric}(\omega)(\tau>\sigma)=\langle\Delta \omega, \omega\rangle(\tau>\sigma)-\frac{1}{2}|\nabla \omega|^{2}(\tau>\sigma)+\frac{1}{2} \Delta^{b}|\omega|^{2}(\tau>\sigma) .
$$

LEMma 4.6. For any combinatorial 1-form $\omega$ on $M$, we have

$$
\begin{array}{r}
\langle\Delta \omega, \omega\rangle(\tau>\sigma)=-\sum_{\left(\mu>\tau^{\prime}\right) ; 2-\text { neighbor }} \frac{w_{\sigma}}{w_{\mu}} \omega_{\sigma}^{\tau} \omega_{\tau^{\prime}}^{\mu}-\sum_{\left(\sigma^{\prime}>\rho\right) ; 2-\text { neighbor }} \frac{w_{\rho}}{w_{\tau}} \omega_{\sigma}^{\tau} \omega_{\rho}^{\sigma^{\prime}} \\
+\sum_{\left(\tau^{\prime}>\sigma\right) ; 0-\text { neighbor }} \frac{\left(w_{\sigma}\right)^{2}}{w_{\tau} w_{\tau^{\prime}}} \omega_{\sigma}^{\tau} \omega_{\sigma}^{\tau^{\prime}}+\sum_{\left(\tau>\sigma^{\prime}\right) ; 0-\text { neighbor }} \frac{w_{\sigma} w_{\sigma^{\prime}}}{\left(w_{\tau}\right)^{2}} \omega_{\sigma}^{\tau} \omega_{\sigma^{\prime}}^{\tau} \\
+\sum_{\left(\mu>\tau^{\prime}\right) ; 2-\text { neighbor }}\left(\frac{\left(w_{\sigma}\right)^{2}}{w_{\tau} w_{\tau^{\prime}}}-\frac{w_{\sigma}}{w_{\mu}}\right) \omega_{\sigma}^{\tau} \omega_{\sigma}^{\tau^{\prime}}+\sum_{\left(\sigma^{\prime}>\rho\right) ; 2-\text { neighbor }}\left(\frac{w_{\sigma} w_{\sigma^{\prime}}}{\left(w_{\tau}\right)^{2}}-\frac{w_{\rho}}{w_{\tau}}\right) \omega_{\sigma}^{\tau} \omega_{\sigma^{\prime}}^{\tau} \\
+(\#\{2-\text { neighbor vector }\}+2)\left(\frac{w_{\sigma}}{w_{\tau}}\right)^{2}\left(\omega_{\sigma}^{\tau}\right)^{2} .
\end{array}
$$

Proof. For a 1-form $\omega$, we have

$$
\begin{aligned}
\left(d^{*} d \omega\right)_{\sigma}^{\tau}= & \sum_{\left(\mu>\tau^{\prime}\right) ; 2-\text { neighbor }} \frac{w_{\mu}}{w_{\tau}}\left(\omega_{\tau}^{\mu}+\omega_{\sigma}^{\tau}-\omega_{\tau^{\prime}}^{\mu}-\omega_{\sigma}^{\tau^{\prime}}\right)+ \\
& \sum_{\left(\sigma^{\prime}>\rho\right) ; 2-\text { neighbor }} \frac{w_{\sigma}}{w_{\rho}}\left(\omega_{\sigma}^{\tau}+\omega_{\rho}^{\sigma}-\omega_{\sigma^{\prime}}^{\tau}-\omega_{\rho}^{\sigma^{\prime}}\right) \\
\left(d d^{*} \omega\right)_{\sigma}^{\tau}=- & -\sum_{\mu>\tau} \frac{w_{\mu}}{w_{\tau}} \omega_{\tau}^{\mu}+\sum_{\tau>\sigma^{\prime}} \frac{w_{\tau}}{w_{\sigma^{\prime}}} \omega_{\sigma^{\prime}}^{\tau}+\sum_{\tau^{\prime}>\sigma} \frac{w_{\tau^{\prime}}}{w_{\sigma}} \omega_{\sigma}^{\tau^{\prime}}-\sum_{\sigma>\rho} \frac{\sigma}{\rho} \omega_{\rho}^{\sigma} .
\end{aligned}
$$


Then the Laplacian of $\omega$ is

$$
\begin{array}{r}
(\Delta \omega)_{\sigma}^{\tau}=-\sum_{\left(\mu>\tau^{\prime}\right) ; 2-\text { neighbor }} \frac{w_{\mu}}{w_{\tau}} \omega_{\tau^{\prime}}^{\mu}-\sum_{\left(\sigma^{\prime}>\rho\right) ; 2-\text { neighbor }} \frac{w_{\sigma}}{w_{\rho}} \omega_{\rho}^{\sigma^{\prime}} \\
+\sum_{\left(\mu>\tau^{\prime}\right) ; 2-\text { neighbor }}\left(\frac{w_{\sigma}}{w_{\tau^{\prime}}}-\frac{w_{\tau}}{w_{\mu}}\right) \omega_{\sigma}^{\tau^{\prime}}+\sum_{\left(\sigma^{\prime}>\rho\right) ; 2-\text { neighbor }} \frac{w_{\tau^{\prime}}}{w_{\sigma}} \omega_{\sigma}^{\tau^{\prime}}+\sum_{\left(\tau>\sigma^{\prime}\right) ; 0-\text { neighbor }} \frac{w_{\tau}}{w_{\sigma^{\prime}}} \omega_{\sigma^{\prime}}^{\tau} \\
\left.+(\#\{2-\text { neighbor vector }\}+2) \frac{w_{\sigma^{\prime}}}{w_{\tau}}-\frac{w_{\rho}}{w_{\sigma}}\right) \omega_{\sigma^{\prime}}^{\tau}\left(\omega_{\sigma}^{\tau}\right) .
\end{array}
$$

Takeing the innner product of $\omega$ and $\Delta \omega$, we have the lemma.

TheOREM 4.7. Let $M$ be a regular quasiconvex cell-complex, and $(\tau>\sigma)$ a vector on $M$. For a combinatorial 1-form $\omega$ on $M$, the Ricci curvature $\operatorname{Ric}(\omega)$ is reprenseted by

$$
\begin{array}{r}
\operatorname{Ric}(\omega)(\tau>\sigma)=(2-\#\{0-\text { neighbor vector of }(\tau>\sigma)\})\left(\frac{w_{\sigma}}{w_{\tau}}\right)^{2}\left(\omega_{\sigma}^{\tau}\right)^{2} \\
+\sum_{\left(\mu>\tau^{\prime}\right) ; 2-\text { neighbor }}\left(\frac{\left(w_{\sigma}\right)^{2}}{w_{\tau} w_{\tau^{\prime}}}-\frac{w_{\sigma}}{w_{\mu}}\right) \omega_{\sigma}^{\tau} \omega_{\sigma}^{\tau^{\prime}}+\sum_{\left(\sigma^{\prime}>\rho\right) ; 2-\text { neighbor }}\left(\frac{w_{\sigma} w_{\sigma^{\prime}}}{\left(w_{\tau}\right)^{2}}-\frac{w_{\rho}}{w_{\tau}}\right) \omega_{\sigma}^{\tau} \omega_{\sigma^{\prime}}^{\tau} .
\end{array}
$$

In particular, with the assumption that the weight of each cell is constant, we have

$$
\operatorname{Ric}(\omega)(\tau>\sigma)=(2-\#\{0-\text { neighbor vector of }(\tau>\sigma)\})\left(\frac{w_{\sigma}}{w_{\tau}}\right)^{2}\left(\omega_{\sigma}^{\tau}\right)^{2} .
$$

Proof. With the above lemma, we have

$$
\begin{aligned}
& 2\langle\Delta \omega, \omega\rangle(\tau>\sigma) \\
& =-\sum_{\left(\mu>\tau^{\prime}\right) ; 2-\text { neighbor }} \frac{w_{\sigma}}{w_{\mu}}\left(\left(\omega_{\sigma}^{\tau}-\omega_{\tau^{\prime}}^{\mu}\right)^{2}-\left(\left(\omega_{\sigma}^{\tau}\right)^{2}-\left(\omega_{\tau^{\prime}}^{\mu}\right)^{2}\right)-2\left(\omega_{\sigma}^{\tau}\right)^{2}\right) \\
& -\sum_{\left(\sigma^{\prime}>\rho\right) ; 2-\text { neighbor }} \frac{w_{\rho}}{w_{\tau}}\left(\left(\omega_{\sigma}^{\tau}-\omega_{\rho}^{\sigma^{\prime}}\right)^{2}-\left(\left(\omega_{\sigma}^{\tau}\right)^{2}-\left(\omega_{\rho}^{\sigma^{\prime}}\right)^{2}\right)-2\left(\omega_{\sigma}^{\tau}\right)^{2}\right) \\
& +\sum_{\left(\tau^{\prime}>\sigma\right) ; 0-\text { neighbor }} \frac{\left(w_{\sigma}\right)^{2}}{w_{\tau} w_{\tau^{\prime}}}\left(\left(\omega_{\sigma}^{\tau}+\omega_{\sigma}^{\tau^{\prime}}\right)^{2}-\left(\left(\omega_{\sigma}^{\tau}\right)^{2}+\left(\omega_{\sigma}^{\tau^{\prime}}\right)^{2}\right)-2\left(\omega_{\sigma}^{\tau}\right)^{2}\right) \\
& +\sum_{\left(\tau>\sigma^{\prime}\right) ; 0-\text { neighbor }} \frac{w_{\sigma} w_{\sigma^{\prime}}}{\left(w_{\tau}\right)^{2}}\left(\left(\omega_{\sigma}^{\tau}+\omega_{\sigma^{\prime}}^{\tau}\right)^{2}-\left(\left(\omega_{\sigma}^{\tau}\right)^{2}+\left(\omega_{\sigma^{\prime}}^{\tau}\right)^{2}\right)-\left(\omega_{\sigma}^{\tau}\right)^{2}\right) \\
& +2(\#\{2-\text { neighbor vector }\}+2)\left(\omega_{\sigma}^{\tau}\right)^{2} \\
& +2 \sum_{\left(\mu>\tau^{\prime}\right) ; 2-\text { neighbor }}\left(\frac{\left(w_{\sigma}\right)^{2}}{w_{\tau} w_{\tau^{\prime}}}-\frac{w_{\sigma}}{w_{\mu}}\right) \omega_{\sigma}^{\tau} \omega_{\sigma}^{\tau^{\prime}}+2 \sum_{\left(\sigma^{\prime}>\rho\right) ; 2-\text { neighbor }}\left(\frac{w_{\sigma} w_{\sigma^{\prime}}}{\left(w_{\tau}\right)^{2}}-\frac{w_{\rho}}{w_{\tau}}\right) \omega_{\sigma}^{\tau} \omega_{\sigma^{\prime}}^{\tau} \\
& =|\nabla \omega|^{2}(\tau>\sigma)-\Delta^{b}|\omega|^{2}(\tau>\sigma)+2(2-\#\{0-\text { neighbor vector }\})\left(\omega_{\sigma}^{\tau}\right)^{2} . \\
& +2 \sum_{\left(\mu>\tau^{\prime}\right) ; 2-\text { neighbor }}\left(\frac{\left(w_{\sigma}\right)^{2}}{w_{\tau} w_{\tau^{\prime}}}-\frac{w_{\sigma}}{w_{\mu}}\right) \omega_{\sigma}^{\tau} \omega_{\sigma}^{\tau^{\prime}}+2 \sum_{\left(\sigma^{\prime}>\rho\right) ; 2-\text { neighbor }}\left(\frac{w_{\sigma} w_{\sigma^{\prime}}}{\left(w_{\tau}\right)^{2}}-\frac{w_{\rho}}{w_{\tau}}\right) \omega_{\sigma}^{\tau} \omega_{\sigma^{\prime}}^{\tau} .
\end{aligned}
$$

Then we have the equation (4.3).

\section{Combinatorial Gauss-Bonnet Theorem}

5.1. Gauss-Bonnet Theorem for Graph. Let $G=(V, E)$ be a finite simple graph, where $V$ is the set of vertexes and $E$ the set of edges. We realize $G$ as 1-dimensional cell complex, i.e. vertexes are 0-cells and edges are 1-cells. 
LEMMA 5.1. Let $v$ and $e$ be a vertex and an edge of $G$ respectivity such that $e>v$. We take a combinatorial 1-form $\omega$ on $G$. Then we have

$$
\operatorname{Ric}(\omega)(e>v)=(2-\operatorname{deg}(v))\left(\frac{w_{v}}{w_{e}}\right)^{2}\left(\omega_{v}^{e}\right)^{2}
$$

where $\operatorname{deg}(v)$ is the degree of $v$.

Proof. 2-cell $f$ such that $f>e, e^{\prime}$, and there are no $(-1)$-cell $\rho$ such that $v, v^{\prime}>\rho$ respectively. Let $v$ and $e$ be a vertex and an edge of $G$ respectivity such that $e>v$. For the definition of 0 -neighbor vector, we find two vectors $\left(e^{\prime}>v\right)$ and $\left(e>v^{\prime}\right)$ such that there are no

Since there are no 2-cells in $G$, the vector $\left(e^{\prime}>v\right)$ is a 0 -neighbor vector for any $e^{\prime}$ which has the vertex $v$ except for the edge $e$. Hence there are exactly $\operatorname{deg}(v)-1$ edges that satisfy the above condition. Since there are no $(-1)$-cells, there is only one vertex $v^{\prime}$ such that $\left(e>v^{\prime}\right)$ is a 0-neighbor vector for $(e>v)$. Then the number of 0-neighbor vectors for $(e>v)$ is $\operatorname{deg}(v)$.

With the definition of a 2-neighbor vecotor, there are not 2-neighbor vectors for the vector $(e>v)$. We have the lemma from the eqation (4.3).

With this lemma, we immediately have the following lemma.

LEMMA 5.2. We take a combinatorial 1 -form $\omega$ on $G$ such that for any vertex $v$

$$
\sum_{e ; e>v}\left(\frac{w_{v}}{w_{e}}\right)^{2}\left(\omega_{v}^{e}\right)^{2}=1
$$

where the sum is taken over all edges e such that $e>v$. Then for any vertex $v$ we have

$$
\sum_{e ; e>v} \operatorname{Ric}(\omega)(e>v)=2-\operatorname{deg}(v)
$$

For a smooth surface the Gauss curvature at a point $p$ equal to the Ricci curvature for a unit vector at $p$. The following definition is an analogue to this fact.

DEFINITION 5.3. We define the Gauss curvature for a vertex $v$ by

$$
g_{v}=2-\operatorname{deg}(v)
$$

Proof of the Theorem 1.1. From the definition of the Gauss curvature, we have

$$
\begin{aligned}
\sum_{v} g_{v} & =\sum_{v}(2-\operatorname{deg}(v)) \\
& =2 \# V-\sum_{v} \operatorname{deg}(v) \\
& =2 \# V-2 \# E \\
& =2 \chi(G) .
\end{aligned}
$$

5.2. Gauss-Bonnet Theorem for 2-complex. Let $M$ be a 2-dimensional quasiconvex cell complex that decomposes a 2-dimensional closed smooth surface.

LEMMA 5.4. Let $v$ and $e$ be a vertex and an edge on $M$ respectivity such that $e>v$. We take a combinatorial 1-form $\omega$ on $M$. Then we have

$$
\operatorname{Ric}(\omega)(e>v)=(4-\operatorname{deg}(v))\left(\frac{w_{v}}{w_{e}}\right)^{2}\left(\omega_{v}^{e}\right)^{2}+\sum_{\left(f>e^{\prime}\right) ; 2-\text { neighbor }}\left(\frac{\left(w_{v}\right)^{2}}{w_{e} w_{e^{\prime}}}-\frac{w_{v}}{w_{f}}\right) \omega_{e}^{v} \omega_{e^{\prime}}^{v},
$$

where $\operatorname{deg}(v)$ is the degree of $v$ and the sum is taken over all 2-neighbor vectors of the vector $(e>v)$. 
Proof. Let $v$ and $e$ be a vertex and an edge on $M$ respectivity such that $e>v$. For the definition of a 0 -neghbor vecotor, we find the vectors $\left(e^{\prime}>v\right)$ and $\left(e>v^{\prime}\right)$ such that there are no 2-cell $f$ such that $f>e, e^{\prime}$ and there are no $(-1)$-cell $\rho$ such that $v, v^{\prime}>\rho$ respectively.

For the edge $e$ there are exactly two faces that have the edge $e$. For exactly two edges $e^{\prime}$ the vectors $\left(e^{\prime}>v\right)$ are not 0 -neghbor vecotors of $(e>v)$. The number of 0-neghbor vecotors of $(e>v)$ is $\operatorname{deg}(v)-3$. Since there are no $(-1)$-cells in $M$, there is only one vertex $v^{\prime}$ such that $\left(e>v^{\prime}\right)$ is a 0 -neighbor vector for $(e>v)$. Then the number of 0-neighbor vecotors for $(e>v)$ is $\operatorname{deg}(v)-2$. We have the lemma from the equation (4.4).

For a vertex $v$ we consider the sum

$$
\operatorname{Ric}(\omega)(v):=\sum_{e ; e>v} \operatorname{Ric}(\omega)(e>v),
$$

where the sum is taken over all edges $e$ that have the vertex $v$. This is a quadratic form for real basises $\left\{\frac{w_{v}}{w_{e}} \omega_{v}^{e}\right\}_{e>v}$. The trace with this basises is $\operatorname{deg}(v)(4-\operatorname{deg}(v))$. For a smooth manifold the scalar curvature is a trace of the Ricci curvature. The next definition is an analogue to this fact.

Definition 5.5. We define the scalar curvature $S(v)$ at a vertex $v$ as

$$
S(v)=\operatorname{trace} \operatorname{Ric}(\omega)(v)=\operatorname{deg}(v)(4-\operatorname{deg}(v)) .
$$

For a smooth surface the scalar curvature is the twice of the Gauss curvature. The next definition is analogue to this fact.

Definition 5.6. We define the Gauss curvature at a vetex $v$ as

$$
g_{v}=\frac{S(v)}{\operatorname{deg}(v)}=4-\operatorname{deg}(v) .
$$

LEMMA 5.7. Let $e$ and $f$ be an edge and a face of $M$ respectivity such that $f>e$. We take a combinatorial 1-form $\omega$ on $M$. Then we have

$$
\operatorname{Ric}(\omega)(f>e)=(4-\operatorname{deg}(f))\left(\frac{w_{e}}{w_{f}}\right)^{2}\left(\omega_{e}^{f}\right)^{2}+\sum_{\left(e^{\prime}>v\right) ; 2-\text { neighbor }}\left(\frac{w_{e} w_{e^{\prime}}}{\left(w_{f}\right)^{2}}-\frac{w_{v}}{w_{f}}\right) \omega_{f}^{e} \omega_{f}^{e^{\prime}},
$$

where $\operatorname{deg}(f)$ is the degree of $f$, that is, the number of edges of $f$ and the sum is taken over the all 2-neighbor vector of the vector $(f>e)$.

Proof. Let $e$ and $f$ be an edge and a face of $M$ respectivity such that $f>e$. For the definition of a 0 -neghbor vecotor, we find two vectors $\left(f^{\prime}>e\right)$ and $\left(f>e^{\prime}\right)$ such that there are no 3-cell $\sigma$ such that $\sigma>f, f^{\prime}$ and there are no 0 -cell $v$ such that $e, e^{\prime}>v$ respectively.

For the edge $e$ there are exactly two faces that have $e$ as an edge. Then for only one face $f^{\prime}$ the vector $\left(f^{\prime}>e\right)$ is a 0 -neighbor vector of $(f>e)$. For edges of the face $f$, exactly two edges $e_{1}, e_{2}$ intersect with the edge $e$, then the two vectors $\left(f>e_{1}\right)$ and $\left(f>e_{2}\right)$ are not 0 -neighbor vectors of $(f>e)$. For the other edges $e^{\prime}$ of the face $f$, the vector $\left(f>e^{\prime}\right)$ is a 0-neighbor vector of $(f>e)$. Then the number of 0 -neighbor vecotors for $(e>v)$ is $\operatorname{deg}(f)-2$. We have the lemma from the eqation (4.4).

For a face $f$ we consider the next sum,

$$
\operatorname{Ric}(\omega)(f):=\sum_{e ; f>e} \operatorname{Ric}(\omega)(f>e),
$$

where the sum is taken over the all edges $e$ contained in the boundary of the face $f$. 
Definition 5.8. We define the scalar curvature $S(f)$ at a face $f$ as

$$
S(f)=\operatorname{trace} \operatorname{Ric}(\omega)(f)=\operatorname{deg}(f)(4-\operatorname{deg}(f)) .
$$

We define the Gauss curvature at a face $f$ as

$$
g_{f}=\frac{S(f)}{\operatorname{deg}(f)}=4-\operatorname{deg}(f) .
$$

If all weights of cells of $M$ are constants, we conclude the following lemma that is an analogue to the smooth surface.

LEMMA 5.9. Let $M$ be a 2-dimensional quasiconvex cell complex that decomposes a 2-dimensional closed smooth surface. We assume that all weights of cells of $M$ are constants.

(1) Let $v$ be a vertex of $M$. We take a combinatorial 1-form $\omega$ on $M$ such that

$$
\sum_{e ; e>v}\left(\omega_{v}^{e}\right)^{2}=1
$$

where the sum is taken over all edges e such that $e>v$. Then we have

$$
\sum_{e ; e>v} \operatorname{Ric}(\omega)(e>v)=4-\operatorname{deg}(v)=g_{v} .
$$

(2) Let $f$ be a face of $M$. We take a combinatorial 1-form $\omega$ on $M$ such that

$$
\sum_{e ; f>e}\left(\omega_{e}^{f}\right)^{2}=1,
$$

where the sum is taken over all edges e such that $f>e$. Then we have

$$
\sum_{e ; f>e} \operatorname{Ric}(\omega)(f>e)=4-\operatorname{deg}(f)=g_{f} .
$$

Proof of Theorem 1.2. We denote $V, E$ and $F$ by the numbers of vertexes, edges and faces in $M$ respectivity.

From the definition of the Gauss curvature, we have

$$
\begin{aligned}
\sum_{v} g_{v}+\sum_{f} g_{f} & =\sum_{v}(4-\operatorname{deg}(v))+\sum_{f}(4-\operatorname{deg}(f)) \\
& =4 V-\sum_{v} \operatorname{deg}(v)+4 F-\sum_{f} \operatorname{deg}(f) \\
& =4 V-2 E+4 F-2 E \\
& =4(V-E+F) \\
& =4 \chi(M) .
\end{aligned}
$$

\section{REFERENCES}

[1] Robin Forman, Bochner's method for cell complexes and combinatorial Ricci curvature, Discrete Comput. Geom. 29 (2003), no. 3, 323-374, DOI 10.1007/s00454-002-0743-x. MR1961004

[2] —, Combinatorial Novikov-Morse theory, Internat. J. Math. 13 (2002), no. 4, 333-368, DOI 10.1142/S0129167X02001265. MR1911862

[3] _ Combinatorial vector fields and dynamical systems, Math. Z. 228 (1998), no. 4, 629-681, DOI 10.1007/PL00004638. MR1644432

[4] _ Morse theory for cell complexes, Adv. Math. 134 (1998), no. 1, 90-145, DOI 10.1006/aima.1997.1650. MR1612391 
[5] Rachel F Arnold, The Discrete Hodge Star Operator and Poincaré Duality, Virginia Polytechnic Institute and State University (2012).

[6] A.T. Lundell and S. Weingram, The Topology of CW Complexes, The university series in higher mathematics, Springer New York, 2012.

[7] Paul Mccorollarymick, Combinatorial Curvature of Cellular Complexes, The University of Melbourne, Department of Mathematics and Statistics (2004).

Kazuyoshi Watanabe

Mathematical Institute

Tohoku University

Sendai $980-8578$

Japan kazuyoshi.watanabe.q5@dc.tohoku.ac.jp 